\title{
Identifying Opportunities to Improve HPV Vaccination in an at-Risk Population
}

\author{
Amanda S Lin ${ }^{1}$, Anjali A Gaikwad ${ }^{1}$, Tran N Le ${ }^{1}$, Christopher D Barr ${ }^{3}$, Lavanya H Palavalli Parsons ${ }^{1}$, Allan R Katz ${ }^{1}$ \\ and Judith A Smith ${ }^{2,4 *}$
}

${ }^{1}$ Department of Obstetrics, Gynecology, \& Reproductive Sciences, UTHealth McGovern Medical School, USA

${ }^{2}$ UTHealth-Memorial Hermann Cancer Center-Texas Medical Center, USA

${ }^{3}$ Department of Statistics, Rice University, USA

${ }^{4}$ Department of Pharmacy, Memorial Hermann-Texas Medical Center, USA

Submission: October 09, 2020; Published: October 14, 2020

*Corresponding author: Judith A Smith BS, PharmD, BCOP, CPHQ, FCCP, FHOPA, FISOPP, Professor \& Director WHIM Research Program, Department of Obstetrics, Gynecology \& Reproductive Sciences, UTHealth McGovern Medical School, 6431 Fannin Street, Rm. 3.152, Houston, TX 77030, USA

\begin{abstract}
Background: To estimate rate of human papillomavirus (HPV) vaccination in an at-risk population tested positive for sexually transmitted diseases (STDs) before and after discussion with provider, assess provider documentation of discussion of the HPV vaccine if not previously fully vaccinated. The HPV infection rates in this at-risk population were also evaluated.

Methods: This retrospective quality improvement research study identified charts by screening for CPT codes for STDs in patients 15 to 38 years of age. The following data were extracted: age at date of service, HPV vaccination status, the number of vaccine doses received, if healthcare provider recommended the HPV vaccination and number of doses received post discussion, and HPV infection history. Descriptive statistics and chi-square analysis were employed as appropriate.

Results: Five hundred patients' charts were identified with 442 charts meeting inclusion criteria. Of the 442 patients, 89 patients (20.1\%) had documentation of HPV vaccination status. Eighteen of the 89 patients had completed the HPV vaccination series. In the 71 patients that had documentation that they had not been or completed vaccination, the provider discussion of the HPV vaccine led to 26 patients initiating vaccination, with 13 completing the series and 13 with incomplete vaccination. Post-discussion, there was a documented $72.3 \%$ improvement in the vaccination rate from $20.2 \%$ to $34.8 \%$ in this population, $(\mathrm{p}<0.05)$. In patients that had documentation of HPV vaccination history, no statistically significant difference in the rate of HPV positive infections was observed: $11 / 50$ (22\%) patients never vaccinated, $5 / 21(23.8 \%)$ patients that had incomplete vaccination and $3 / 18(16.7 \%)$ patients that had completed vaccination.
\end{abstract}

Conclusion: Improvement is needed in HPV vaccination documentation in gynecology offices. Discussion to receive the HPV vaccine in at risk patients improved compliance with HPV vaccination rates. Additional evaluation is needed on the long-term efficacy of HPV vaccination in an at-risk/sexually active patient population.

Keywords: HPV; STDs; HPV vaccine

Abbreviations: HPV: Human Papilloma Virus; STD: Sexually Transmitted Disease; FDA: Food and Drug Administration; ACIP: Advisory Committee on Immunization Practices; UT University of Texas; CIN1: Cervical intraepithelial Neoplasia Grade 1; LEEP: Loop Electrosurgical Excision Procedure (LEEP)

\section{Introduction}

The human papillomavirus (HPV) is the most prevalent sexually transmitted disease (STD) worldwide, with $50-80 \%$ of sexually active individuals contracting an infection in their lifetime [1]. HPV was first recognized as the cause of the majority of cervical cancer cases in 1980. It is now also known to cause at least five other epithelial surface cancers, including anal, oropharyngeal, penile, vaginal, and vulvar cancers. Globally in 2012, about 580,000 cancer diagnoses in women and 60,000 cancer diagnoses in men were a result of an HPV infection. 1 To help prevent this, the HPV vaccine was developed and first licensed in 2006 by the U.S. Food and Drug Administration (FDA) as a quadrivalent HPV (4vHPV) vaccine effective against HPV 6/11/16/18 strains 


\section{Global Journal of Reproductive Medicine}

given as a three-dose series. Its successors, the bivalent vaccine and the nine-valent vaccine, were released in the U.S. in 2009 and 2014 respectively [2]. As of 2016, the U.S. Advisory Committee on Immunization Practices (ACIP) recommends a two-dose schedule for ages 9 through 14 years, with the second dose administered 6-12 months after the first dose (0, 6-12-month schedule). Routine vaccination is recommended at age 11 or 12 years. For those starting vaccination on or after their 15 th birthday, a threedose schedule is recommended through age 26 . The second dose should be given one to two months after the first dose, and the third dose should be given six months after the first dose [3]. In October of 2018, the FDA approved the nine-valent HPV vaccine (Gardasil-9, Merck \& Co., INC, West Point, PA) for expanding use to men and women aged 27 through 45 years [4]. However, the ACIP is still reviewing the available data and has not yet recommended expanded use to these groups [5]. Healthy People 2020 has established a goal for $80 \%$ of all adolescents to have completed the recommended number of HPV vaccine doses by age 13 to 15 years. However, the national rate has lagged behind that, with Texas having significantly lower rates compared to the national average. In 2016, fewer than half $(49.3 \%)$ of Texas adolescents have received one dose of HPV vaccine, which is $10.6 \%$ lower for females and $11.7 \%$ lower for males compared to U.S. vaccination rates. Additionally, only about one-third (32.9\%; 39.7\% of females; $26.5 \%$ of males) have completed their HPV vaccinations. This ranks Texas at 47 th out of 50 states plus the District of Columbia for HPV vaccination completion, with only Wyoming, Mississippi, South Carolina, and Utah having lower up-to-date HPV vaccination rates [6]. Studies have shown that HPV is commonly a co-infection with other STDs [7]. Different studies have shown varied rates of co-infection, ranging from $20 \%$ to $69 \%$ of HPV positive individuals being co-infected with another STD. [8-10]. Therefore, those who test positive for an STD are an at-risk population for potential exposure to HPV infections. HPV is transmitted only through intimate, direct skin to skin contact. It is not airborne or transmitted by casual contact. One can conclude that patients with no sexual/intimate contact would have no risk for contracting HPV infections. However, previous studies examining the presence of HPV subtypes and antibody titers have been limited by patient populations with no or unknown sexual exposure. While past activity may have been documented, sexual activity during the studies was not documented or at least included in published reports and, for that reason, there was unknown exposure to HPV infections [11-14].

Based on this premise, this quality improvement study attempted to obtain real-life data in those who are sexually active and have a real potential risk of exposure to HPV and contracting HPV infections. This was done by evaluating patients that have confirmed positive test for that can only be contracted by direct contact. The hypothesis of this quality improvement study was that there is low rate of vaccination rate in the at-risk population and an opportunity to improve HPV vaccination rates at the time of a new STD diagnosis. The first objective was to determine the rate of vaccination in an at-risk population before and after discussion with provider and then assess the consistency of documentation of providers offering education and recommendation to at-risk patients to receive the HPV vaccine if not previously vaccinated or incompletely vaccinated. Since there is limited data available on the vaccine efficacy in this defined at-risk population, a secondary objective was to observe the HPV infection status in relationship to previous HPV vaccination. The importance of this study is to improve the scientific community's knowledge on the value of the quality and consistency of HPV vaccination documentation and to generate data to support future studies to evaluate long-term efficacy of the HPV vaccines in an at-risk patient population.

\section{Methods}

This retrospective study was reviewed and approved to proceed by the University of Texas (UT) Health Sciences Center at Houston institutional review board as a quality improvement research study. The data were obtained from the UT Physicians Women's Center clinics. In this quality improvement study, the first 500 patient charts were identified by screening for billing CPT codes, ICD-9 and ICD-10 as indicated, for the following STDs: herpes, genital herpes, gonorrhea, chlamydia, trichomonas, cancroid, urethritis, bacterial vaginosis, and molluscum contagiosum, which correlated with dates between December 2015 and November 2018. Hepatitis and HIV were excluded since these STDs are not exclusively contracted by sexual contact. Once patient charts were identified with STD diagnosis, only charts for patients that were between the ages of 15 and 38 years of age were included. Women older than 38 would not have been eligible to receive the vaccine when first released in 2006, and the practice does not see patients younger than [15]. Each chart was reviewed and the following data were extracted: age at date-of-service, HPV vaccination status, if previously vaccinated the number of doses received, and if never/incompletely vaccinated then evaluation of if healthcare provider discussed/recommended the vaccine. If the provider had discussion/recommendation to get the HPV vaccine, then number of doses received post-discussion was documented. Finally, occurrence of HPV infections was documented for all patients. Documentation was determined based on entries into each patient chart's vaccine record and/or physician's notes indicating discussion of the patient's vaccine status. Vaccine discussion occurred when the provider delivered counseling to recommend receiving or complete the series. Vaccine status was determined using the 2016 ACIP guidelines with the number of doses received before and after the date-of-encounter of vaccine discussion. HPV infection status was positive if the patient ever had documentation of a positive HPV test or had a history of cervical intraepithelial neoplasia grade 1 (CIN1) or greater, loop electrosurgical excision procedure (LEEP), or cervical cryotherapy. All data were entered into a de-identified, secured electronic data base. An independent review of the data was conducted to confirm data collection accuracy. Data analysis was completed to determine the consistency of vaccination documentation, 
prevalence of HPV infection, rate of discussion of vaccination, and change in vaccination rate before and after discussion. Descriptive statistics were completed to describe the results. Chi-square analysis was used to evaluate differences in rates of HPV infections and vaccination rates.

\section{Result}

Five hundred charts associated with recent STD diagnosis were identified from initial CPT code screening. There were 442 patient charts meeting inclusion criteria. Of the 442 patients, 89 patients $(20.1 \%)$ had documentation of HPV vaccination and 353 patients $(79.9 \%)$ did not. There was documentation by the provider of a discussion regarding HPV vaccine status for 74 patients $(16.7 \%)$ and no documentation of discussion for 368 patients (83.3\%). Additionally, HPV infection status was not documented in 254 patients (57.5\%), positive in 121 (27.4\%), and negative in 67 (15.2\%). Of the 89 patients with documentation of vaccination status, 18 had completed the series prior to diagnosis of STD, making $20.2 \%$ the baseline vaccination rate. Therefore, 71 had the opportunity for discussion of vaccination. Of these 71 patients, 24 ultimately were not vaccinated - with
11 of those patient's ineligible for vaccination based on age at time-of-service and 13 declining vaccination. However, the provider discussion of the HPV vaccine led to 26 of the 71 patients initiating vaccination - with 13 completing the series and 13 with incomplete vaccination. Post-discussion, the vaccination rate was $34.8 \%$, resulting in a statistically significant increased rate of vaccination from baseline of 18 vaccinated to 31 vaccinated or a $72.3 \%$ improvement, $(\mathrm{p}<0.05)$. In addition, with exclusion of patients with undocumented HPV infection status, the rate of HPV coinfection in this sexually active patient population with known STD exposure was $64.4 \%$. It was determined that 19 patients had both a positive HPV infection status and documentation of HPV vaccination. Of these 19 patients, there was no statistically significant difference in the observed rates of HPV infections in those patients that had been previously vaccinated compared those who were never or incompletely vaccinated. This included 11 out of 50 patients who had never been vaccinated (22\%), 5 out of 21 patients who had incomplete vaccination (23.8\%), and 3 out of 18 patients who had completed vaccination (16.7\%), p>0.05 as illustrated in Table 1.

Table 1: Pre-Discussion HPV Vaccination Status of HPV Positive Patients, Excluding Patients with Undocumented Vaccine Status.

\begin{tabular}{|c|c|c|}
\hline Vaccination Status & $\begin{array}{c}\text { Number of Patients with Documented HPV } \\
\text { Vaccination Status [N=89] }\end{array}$ & Number Patients with Documented HPV Infection [N (\%)] \\
\hline Never Vaccinated & 50 & $11(22.6)$ \\
\hline Incomplete Vaccination & 21 & $5(23.8)$ \\
\hline Complete Vaccination & 18 & $3(16.7)$ \\
\hline
\end{tabular}

\section{Discussion}

Similar to the low HPV vaccination rates nationwide, a low rate of vaccination was observed in this at- risk population from clinics in a large metropolitan city. However, this study demonstrated that simply taking a history of a patient's HPV vaccination with provider education and discussion of vaccination significantly improved completion of HPV vaccination series in this at-risk population. It is possible this discussion occurred more often than observed, but in absence of appropriate documentation in the medical chart, similar to billing requirements, it was assumed it did not happen. Hence, this study also demonstrated the importance of documentation of physician recommendations in the medical record. An interesting, yet concerning observation was that there was no statistically significant difference in the rate of HPV infections in patients that completed the HPV three shot vaccination series compared to those patients who were never vaccinated or had history of incomplete HPV vaccination. The current literature is limited by the ability to evaluate HPV vaccine efficacy truly and prospectively. It would be unethical to intentionally expose patients to HPV. However, the failure to confirm sexual activity and potential risk of exposure to sexually transmitted diseases such as HPV, is a caveat to consider in evaluating current data. A study similar to the current study did examine sexually active adolescents and also found a high rate of HPV infection similar to the current study [15]. Since the sexual activity was self-reported and the likelihood of HPV exposure still was not as certain compared to the current study that patients had known STD that could only be acquired from direct sexual activity [15].

Other studies have not routinely monitored or documented sexual activity thus the risk for exposure to HPV was unknown [11-17]. Historically, the initial clinical trials evaluating the quadra-valent and nine-valent HPV vaccines have reported to have over a $95 \%$ response rate in patient population 9 to 26 years [14]. While this impressive response rate is the focus of HPV vaccination educational materials, there is long-term follow up data suggesting the efficacy could be as low as $60 \%$ for the quadra-valent HPV vaccine [18]. Another caveat to consider is that since the introduction of the nine valent vaccine, the HPV assay used for screening for HPV has converted from the historical HPV DNA assay to the HPV RNA assay. It has been demonstrated that the HPV RNA assay is less reliable in detection of latent HPV 


\section{Global Journal of Reproductive Medicine}

infections $[14,18,19]$ Thus the true rate of latent HPV infections might be underreported, reflecting a higher efficacy of the ninevalent vaccine compared to the quadra-valent vaccine. This study examined a patient population confirmed to be sexually active through STD diagnoses and demonstrated the potential benefits HPV vaccination counseling effectiveness to engage those not previously vaccinated to complete the HPV vaccination series up to 15 years after being eligible to be vaccinated. One of the reasons that parents often use to decline HPV vaccination for their children was concern of increased sexual activity, so efforts were made to closely evaluate the incidence of STDs in patient's postHPV vaccination. Two recent comprehensive studies did confirm that patients that received the HPV vaccination did not have an increased incidence of STDs, which was reassuring. However, it was surprising neither study there reported the impact receiving the HPV vaccine on the rate of HPV infection compared to those not vaccinated $[16,17]$. Although the number of charts was small in this current study, the observation of similar rates of HPV infections in the patients that had documentation of completing the HPV vaccination compared to those that had incomplete or never vaccinated status is concerning and merits future studies to better define the HPV vaccine's long-term efficacy in sexually active patients with a higher risk of exposure to HPV infections. This study illustrated how few of these patients had documentation of HPV vaccination or had counseling regarding completing vaccination. By simply initiating discussion of vaccination and taking a history of prior HPV vaccinations, providers were able to significantly increase compliance with HPV vaccine. The large number of patients with undocumented HPV vaccination reflected how important it is to document this routinely in a patient's history to improve inter-physician communication and overall patient safety and satisfaction. There were likely many missed opportunities for the provider to intervene and potentially improve compliance with HPV vaccination in this the sexually active population. Several opportunities for improvement were identified for the quality of documentation if/when patients received the HPV vaccination as well as benefits of education in an at-risk population, which may prevent both HPV infection and ultimately HPV-related cancers. There were inconsistencies in both how often and how documentation of HPV vaccination was observed in the overwhelming majority of charts reviewed.

To improve documentation of HPV vaccination history, tools such as clinical templates or checklists should be developed. For example, within the template all physicians would be prompted to complete the HPV vaccination history when taking a patient's gynecologic history that would include all relevant information regarding HPV vaccination. Another under-utilized resource is the state-required vaccination record that can help accurately transfer patients' HPV vaccination history, especially in the transition from the pediatrician office to the gynecology office setting [20]. Other uses of technology, through for instance phone applications, would allow patients to accurately track their vaccination history or allow clinics to efficiently transfer vaccination data into electronic medical records. This information should be routinely incorporated into patient intake workflows for all visit types, including new patient visits and obstetrical visits [21]. Another suggestion is to develop and implement an "STD management clinical pathway" once a diagnosis of a STD is made to navigate a patient through treatment of their diagnosed STD and screening/ treatment for other STDs. Both patients diagnosed with an STD and those present for other visit types should additionally navigate through an "HPV management clinical pathway", to obtain HPV immunization history and administer applicable vaccination. The clinical pathway is illustrated in Figure 1. This study had inherent limitations from its retrospective structure. First, the extent of data that could be obtained was limited by what had been accurately documented by the physician and if the vaccination records had been updated. For example, the vast majority of charts had undocumented vaccination status and HPV infection status, and screening for HPV infection does not occur routinely for those under age 30 , making it difficult to draw conclusions about vaccine efficacy in these patients. Ultimately only identifying 89 patient charts with HPV vaccination out of the 442 charts included limited the impact of the observation of no statistical difference regardless of HPV vaccination status between the rate of HPV co-infection rates in these patients with current diagnosis of STD. However, it does at least raise the question of the long-term HPV vaccine efficacy in sexually active/at risk patient population. One of the study strengths is that it specifically focused on only patients with known STD diagnosis and hence confirmed to be sexually active and with more likely to have potential exposure to HPV infections.

\section{Conclusion}

In a sexually active patient population with known STD infection and hence are at-risk for potential exposure to HPV, it is especially important to identify opportunities to reduce these patients' risk of contracting HPV. The introduction of the HPV vaccine has been exciting and the potential to prevent cancer has gained enthusiastic support. However, this study suggests there are still many opportunities to improve the utilization of the HPV vaccine. First better interprofessional communication is needed to improve HPV vaccination documentation in the transition from practice to practice, such as from the pediatric setting to gynecology offices, between primary care offices, and with retail pharmacies that now provide HPV vaccination in many states. Implementing tools to facilitate discussion of HPV vaccination can improve compliance with HPV vaccination, especially with STD-confirmed patients with greater likelihood to be at-risk for exposure to HPV infections. While only a small number was identified in this study, the observation of a similar rate of HPV infections in women that had previously completed HPV vaccination compared to those women who had not been vaccinated or had "incomplete" vaccination in this sexually active 


\section{Global Journal of Reproductive Medicine}

patient population was unexpected and somewhat alarming. Reflecting the initial reported efficacy of the nine-valent vaccine and even the quadrivalent vaccine warrants additional evaluation in a larger patient population with a known risk for potential exposure to HPV infections.

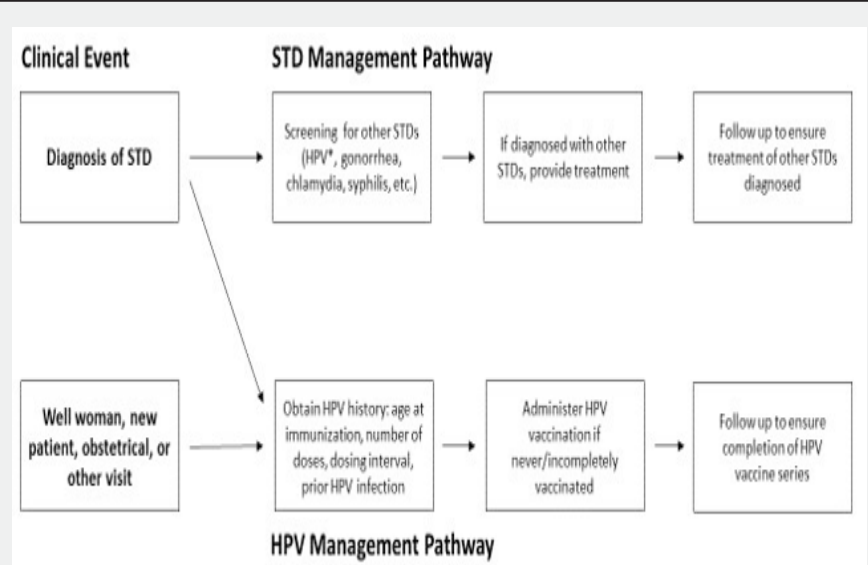

Figure 1: Clinical Pathway for STD and HPV Management.

\section{Funding}

This research was an unfunded, independent investigatorinitiated study.

\section{References}

1. Laurent J, Luckett R, Feldman S (2018) HPV vaccination and the effects on rates of HPV-related cancers. Current Problems in Cancer 42 :493506

2. Pils S, Joura E (2015) From the monovalent to the nine-valent HPV vaccine. Clinical Microbiology and Infection 21: 827-33.

3. Meites E, Kempe A, Markowitz L (2016) Use of a 2-dose schedule for human papillomavirus vaccination-updated recommendations of the advisory committee on immunization practices. Morbidity and Mortality Weekly Report 65: 1405-1408.

4. US Food \& Drug Administration. FDA approves expanded use of Gardasil 9 to include individuals 27 through 45 years old.

5. The American College of Obstetricians and Gynecologists. Practice advisory: FDA approval of 9-valent HPV vaccine for use in women and men age, pp. 27-45.

6. (2019) Vaccine for Use in Women and Men Age 27 to 45 ?

7. Nehme E, Patel D, Oppenheimer D, Elerian N, Lakey D (2017) Missed opportunity: human papillomavirus vaccination in Texas.

8. Dempsey A (2008) Human papillomavirus: The usefulness of risk factors in determining who should get vaccinated. Reviews in Obstetrics and Gynecology 1: 122.

9. Shim HS, Noh S, Park AR, Lee YN, Kim JK, et al. (2010) Detection of sexually transmitted infection and human papillomavirus in negative cytology by multiplex-PCR. BMC Infectious Diseases 10: 284.

10. André LP de Abreu M (2016) Association of human papillomavirus, Neisseria gonorrhoeae and Chlamydia trachomatis co-infections on the risk of high-grade squamous intraepithelial cervical lesion. American Journal of Cancer Research 6(6): 1371-1383.
11. Menezes L, Pokharel U, Sudenga S, Botha M, Zeier M, et al. (2017) Patterns of prevalent HPV and STI co-infections and associated factors among HIV-negative young Western Cape, South African women: the EVRI trial. Sexually Transmitted Infections 94: 55-61.

12. Nygård M, Saah A, Munk C, Tryggvadottir L, Enerly E, et al. (2015) Evaluation of the long-term anti-human papillomavirus 6 (HPV6), 11,16 , and 18 immune responses generated by the quadrivalent HPV vaccine. Clinical and Vaccine Immunology 22: 943-648.

13. Naud P, Roteli Martins C, De Carvalho N, Teixeira J, de Borba P, et al. (2014) Sustained efficacy, immunogenicity, and safety of the HPV-16/18 AS04-adjuvanted vaccine. Human Vaccines \& Immunotherapeutics 10: 2147-2162.

14. Einstein M, Takacs P, Chatterjee A, Sperling R, Chakhtoura N, et al. (2014) Comparison of longterm immunogenicity and safety of human papillomavirus (HPV)-16/18 AS04-adjuvanted vaccine and HPV-6/11/16/18 vaccine in healthy women aged 18-45 years: Endof-study analysis of a Phase III randomized trial. Human Vaccines \& Immunotherapeutics 10: 3435-3445.

15. Villa LL, Costa RLR, Petta CA, Andrade RP, Paavonen J, et al. (2006) High sustained efficacy of a prophylactic quadrivalent human papillomavirus types 6/11/16/18 LI virus-like particle vaccine through 5 years of follow-up. Britis Journal of Cancer 95(11): 1459-1466.

16. Michala L, Argyri E, Tsimplaki E, Tsitsika A, Bakoula C, et al. (2012) Human papilloma virus infection in sexually active adolescent girls. Gynecologic Oncology 126: 207-210.

17. Bednarczyk RA, Davis R, Ault K, Orenstein W, Omer SB (2012) Sexual activity-related outcomes after human papillomavirus vaccination of 11- to 12-year-olds. Pediatrics 130: 798-805.

18. Jena AB, Goldman DP, Seabury SA (2015) Incidence of sexually transmitted infections after human papillomavirus vaccination among adolescent females. JAMA Internal Medicine 175: 617-623.

19. (2019) Texas Health and Human Services. Imm Trac2, the Texas Immunization Registry.

20. (2019) National Vaccine Information Center. How effective is HPV vaccine? 
21. Cotton S, Brown RE, Nugent EK, Robazetti SC, Berens PD, et al. (2018) Quality improvement to demonstrate the lack of reliability of the human papillomavirus mRNA assay to identify women with latent human papillomavirus infections. Obstetrics \& Gynecology 131: 68167.

Your next submission with Juniper Publishers
will reach you the below assets
- Quality Editorial service
- Swift Peer Review
- Reprints availability
- E-prints Service
- Manuscript Podcast for convenient understanding
- Global attainment for your research
- Manuscript accessibility in different formats
( Pdf, E-pub, Full Text, Audio)
- Unceasing customer service
Track the below URL for one-step submission
https://juniperpublishers.com/online-submission.php

